The 2004 IEEE Asia-Pacific Conference on

Circuits and Systems, December 6-9, 2004

\title{
DESIGN AND SIMULATION OF A MIMO OFDM BASEBAND TRANSCEIVER FOR HIGH THROUGHPUT WIRELESS LAN
}

\author{
Chi-Yeh Yu, Zih-Yin Ding, and Tzi-Dar Chiueh \\ Graduate Institute of Electronics Engineering \\ and Department of Electrical Engineering \\ National Taiwan University, Taipei, Taiwan 10617 \\ chiueh@cc.ee.ntu.edu.tw
}

\begin{abstract}
This paper proposes a MIMO OFDM baseband transceiver design for the next-generation high-throughput wireless LAN using two transmit antennas and two receive antennas. A MIMO OFDM receiver with algorithms for timing and frequency synchronization, tracking, channel estimation, and MIMO detection is designed and implemented in software. Simulation results show that the proposed receiver is capable of transmission with a data rate that is twice that of the current IEEE 802.11a wireless LAN standard.
\end{abstract}

\section{INTRODUCTION}

One of the main objectives of current wireless communication research is to increase the link throughput in a spectrallyefficient and reliable manner. Multiple-input multiple-output (MIMO) techniques have the potential of increasing the transmission rate by several folds [1]. In general, there are two types of MIMO techniques: (a) rate maximization schemes such as vertical Bell Laboratories Layered Spare-Time code (V-BLAST) [2] and (b) diversity maximization schemes such as space-time block code (STBC) [3]. The former can achieves a higher data rate, while the latter gains more diversity. Both types of MIMO techniques were developed originally for flat fading channels, and lately have been applied to orthogonal frequency division multiplexing (OFDM) technology used in frequency-selective fading channels.

In this paper, we propose a baseband transceiver capable of doubling the data rate of IEEE 802.11 a standard with two transmit and two receive antennas. The data rate ranges from $6 \times 2$ Mbps to $54 \times 2$ Mbps. A new packet format based on the original format is designed for accommodating MIMO channel estimation. Moreover, several synchronization algorithms for MIMO OFDM receiving are implemented. Functional simulation results of the proposed receiver establish the validity of it effectiveness in high-throughput wireless LAN applications.

The organization of the paper is as follows. Section 2 describes the system as well as the packet format. Section 3 gives the receiver architecture and explains respective algorithms. In Section 4 the simulation results are provided. Finally, conclusions are drawn in Section 5.

\section{SYSTEM DESCRIPTION AND PACKET FORMAT}

The proposed MIMO OFDM system is illustrated in Fig. 1. At the transmitter, data symbols are first processed by MIMO transmission techniques. The MIMO transmission will arrange symbols in $2 \times 2$ VBLAST or in $2 \times 2$ STBC according to the adopted data rate. The processed data symbols are then sent to an IFFT operation and a cyclic prefix (CP) is inserted. At the receiver, $\mathrm{CP}$ is removed, and the data symbols are recovered after an FFT operation. Finally, data symbols are equalized, and then detected.

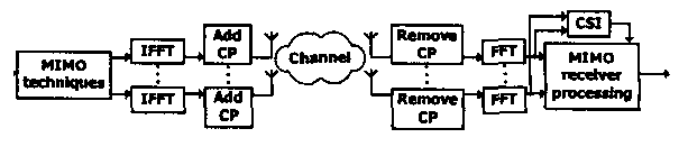

Fig. 1. MIMO OFDM system model.

Physical layer key parameters used in the proposed MIMO OFDM transceiver are listed in Table 1. Note that all are compatible with the IEEE 802.11a standard. Additional double data rates newly provided by the MIMO techniques are listed in Table 2. Note that VBLAST-OFDM can achieve a higher throughput due to parallel transmission, while STBC-OFDM gains more diversity by coding over different branches.

The new MIMO OFDM packet format is shown in Fig. 2. The preamble consists of 10 short symbols, denoted as $t_{1}$ to $t_{10}$, and four long symbols, denoted as $T_{1}$ to $T_{4}$. The preamble is followed by the SIGNAL field and DATA symbols. Identical to the IEEE 802.11a standard, the short preamble utilizes only 12 out of 52 subcarriers, whose indices are a multiple of 4 , and hence results in a periodicity of $T_{F F T} / 4=0.8$ us. The long preamble also reuses the IEEE 802.11a design, with 52 subcarriers (excluding a zero value at DC) modulated by a fixed \pm 1 sequence. The period of long preamble is $T_{F F T}=3.2$ us. Two periods are preceded by a guard interval (GI) of 1.6 us. For MIMO channel estimation, there are four periods and the last two periods and the associated GI in one of the transmitter are inverted. 


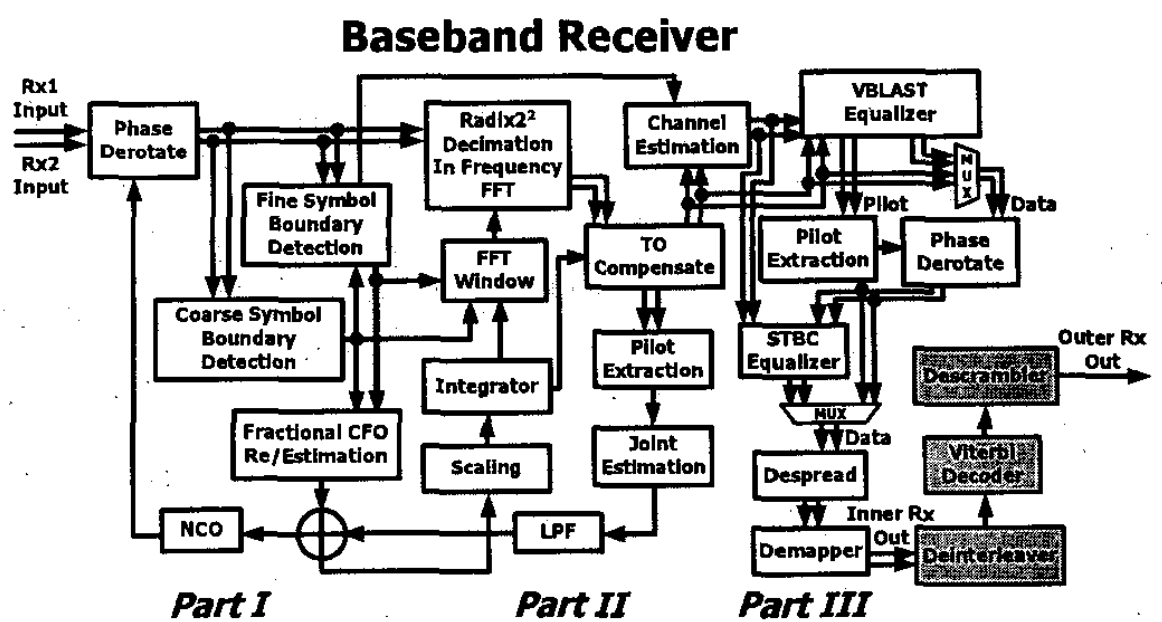

Fig. 3. Block diagram of the proposed MIMO OFDM receiver.

Table 1. Physical layer key parameters.

\begin{tabular}{|c|c|}
\hline Modulation type & $\begin{array}{c}\text { BPSK, QPSK, } \\
\text { 16QAM, 64QAM }\end{array}$ \\
\hline FFT size & 64 \\
\hline Subcarrier in use & $\begin{array}{c}52 \\
\text { (4 for pilot, 48 for data) }\end{array}$ \\
\hline Bandwidth : & $\begin{array}{c}20 \mathrm{MHz} \\
\text { (Occupied } 16.6 \mathrm{MHz})\end{array}$ \\
\hline Subcarrier spacing & $312.5 \mathrm{KHz}(=20 \mathrm{MHz} / 64)$ \\
\hline FFT period & $3.2 \mathrm{us}(=1 / 312.5 \mathrm{KHz})$ \\
\hline Guard interval duration & $0.8 \mathrm{us}$ \\
\hline OFDM symbol duration & $4.0 \mathrm{us}(=3.2+0.8 \mathrm{us})$ \\
\hline
\end{tabular}

Table 2. Achievable data rates

Table 2. Achievable data rates.
\begin{tabular}{|c|c|c|c|}
\hline $\begin{array}{c}\text { Data rate } \\
(\mathrm{Mbps})\end{array}$ & $\begin{array}{c}\text { QAM } \\
\text { Constellation }\end{array}$ & $\begin{array}{c}\text { Code } \\
\text { rate }\end{array}$ & $\begin{array}{c}\text { MIMO } \\
\text { transmission }\end{array}$ \\
\hline 108 & 64 QAM & $3 / 4$ & $2 \times 2$ VBLAST \\
\hline 96 & 64 QAM & $2 / 3$ & $2 \times 2$ VBLAST \\
\hline 72 & 64 QAM & $1 / 2$ & $2 \times 2$ VBLAST \\
\hline 48 & 16 QAM & $1 / 2$ & $2 \times 2$ VBLAST \\
\hline 24 & QPSK & $1 / 2$ & $2 \times 2$ VBLAST \\
\hline 12 & QPSK & $1 / 2$ & $2 \times 2$ STBC \\
\hline
\end{tabular}

\section{RECEIVER FUNCTION AND ARCHITECTURE}

The overall receiver functional block diagram is shown in Fig. 3. We divide the receiver into three parts: Part I is responsible for timing and frequency synchronization in the acquisition mode; Part II is intended for residual carrier frequency offset (CFO) tracking and residual timing offset (TO) tracking; and Part III deals with channel estimation and data recovery.

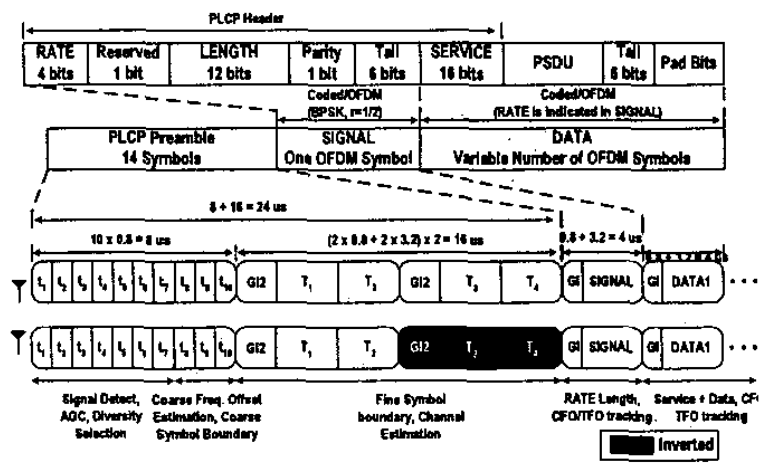

Fig. 2. Proposed MIMO OFDM packet format.

\subsection{Synchronization in the acquisition mode}

Timing and frequency synchronization are performed in the acquisition mode. These tasks includes signal detection, fractional CFO estimation, coarse symbol boundary detection [4], and fine symbol boundary detection. To allow time for automatic gain control (AGC) and energy detection, we assume only the last five short preamble periods are available for synchronization.

Step 1) Signal detection and coarse symbol boundary detection - Due to the periodicity of the short preamble, we use the normalized delay correlation $M(k)$ to detect the existence of short preambles, and averaging the results from the two receive branches,

$$
\begin{gathered}
P(k)=\sum_{q=1}^{2} \sum_{n=k-L+1}^{k} r_{q}(n) r_{q}^{*}(n-d), \\
R(k)=\sum_{q=1}^{2} \sum_{n=k-L+1}^{k}\left|r_{q}(n)\right|^{2} \\
M(k)=\frac{|P(k)|^{2}}{(R(k))^{2}}
\end{gathered}
$$


where $k$ is the time index, $q$ is the receive antenna index, $L$ is the summation length of delay correlation, $d$ is the delay, and $r_{q}(n)$ denotes the received $n$-th sample from the $q$-th antenna. $P(k), R(k)$, and $M(k)$ represent the delay correlation, signal energy, and normalized delay correlation, respectively.

For practical reasons, we approximate the delay correlation by

$$
P(k) \simeq \sum_{n=k-L+1}^{k}\left(r_{1}(n)+r_{2}(n)\right)\left(r_{1}^{*}(n-d)+r_{2}^{*}(n-d)\right)
$$

After applying moving average to smooth the delay correlation, the coarse symbol boundary is determined at the falling edge of the moving average $P_{\text {ave }}(k)$,

$$
P_{a v e}(k)=\frac{1}{w} \sum_{n=k \rightarrow w+1}^{k} P(n)
$$

where $w$ is window size for moving average.

Step 2) Fractional CFO estimation - Carrier frequency offset between the transmitter and the receiver will reflect on the phase of the received time-domain signal. We can average the delay correlation and calculate the corresponding phase shift to estimate the CFO.

$$
\Delta \hat{f}=\frac{\hat{\phi}}{2 \pi d T_{s}}
$$

where $\phi$ is the phase of $P(k)$ and $T_{s}$ is the sampling interval.

Step 3) Fine symbol boundary detection - The long preamble has a PN-code like time-domain characteristic, so we match the received signal to several time-shifted versions of the long preamble waveform by a bank of correlators. The outputs of the correlator bank thus approximate the channel impulse response, from which the first-arrival path can be determined. Fig. 4

The synchronization process simulation is illustrated in

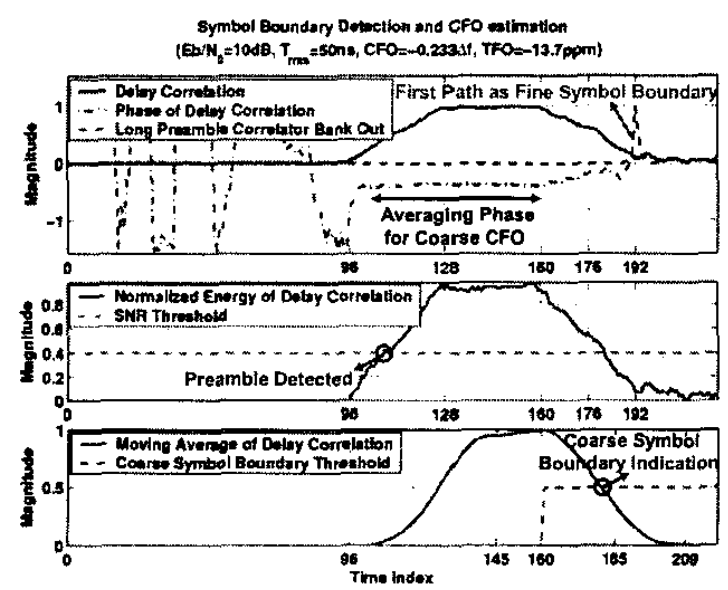

Fig. 4. Synchronization process when receiving the preambles of a packet.

\subsection{CFO/TO tracking}

Though residual CFO/TO are small after fractional CFO compensation, the accumulated phase rotation can still deteriorate system performance as time goes by. Consequently, we need a CFO/TO tracking loop to dynamically compensate such signal phase drift.

To estimate the residual CFO/TO, we adopt the joint weighted least squares (WLS) algorithm [5]. Because we have double the number of pilots from two branches, the accuracy can be enhanced when compared to the case with single receiving antenna. Assume there is only one oscillator in the receiver, WLS algorithm can be simplified, and we can estimate the CFO first and scaling the CFO by a constant to get the TO. For ICI level and simplicity, we choose compensate the CFO in the time domain and compensate the TO in the frequency domain. To limit the noise, we introduce a first-order low-pass proportional-integral (PI) filter in the tracking loop. Finally, as the sampling timing overflows or underflows, we shift the FFT window forward or backward by one sample so as to avoid inter-symbol interference.

\subsection{Channel estimation and data recovery}

The long preamble is especially designed for MIMO channel estimation. By inverting the last two periods of the long preamble in one of the transmitter, we can adopt the STBC combining method to estimate the channel as in [4]. The two extra periods of long preamble can also improve the channel estimation accuracy.

For a certain subcarrier $i$, the received symbol matrix $\mathbf{R}$ can be expressed in terms of the transmit symbol matrix $\mathbf{S}$, the channel matrix $\mathbf{H}$, and the noise matrix $\mathbf{N}$ as

$$
\mathbf{R}_{i, 2 \times 2}=\mathbf{S}_{i, 2 \times 2} \mathbf{H}_{i, 2 \times 2}+\mathbf{N}_{i, 2 \times 2} .
$$

Channels can then be estimated by

$$
\mathbf{H}_{i, 2 \times 2}=\mathbf{S}_{i, 2 \times 2}^{-1} \mathbf{R}_{i, 2 \times 2} .
$$

In low SNR case, to further enhance the channel estimation performance, we add a frequency-domain filter to smooth the estimation result. This filter is designed in such a way that its time-domain waveform is a window of proper shape and length to extract the channel impulse response and reject as much noise as possible [6]. Mean square errors of the estimated channel responses using the filtered and the unfiltered schemes are shown in Fig. 5. The improvements of the filtered channel estimation method is quite apparent. Using the estimated channel responses, we can detect the VBLAST OFDM signal and STBC OFDM signal as described in [2] and [3], respectively.

\section{SIMULATION RESULTS}

The whole MIMO OFDM receiver is implemented in software and the simulated packet error rates (PER) of the demodulated signals are depicted in Fig. 6 . In this simulation, we use the IEEE 802.11 task group n channel model D, which models a typical office environment. The center frequency is set to be $5320 \mathrm{MHz}$, and the CFO and the TO are both set 


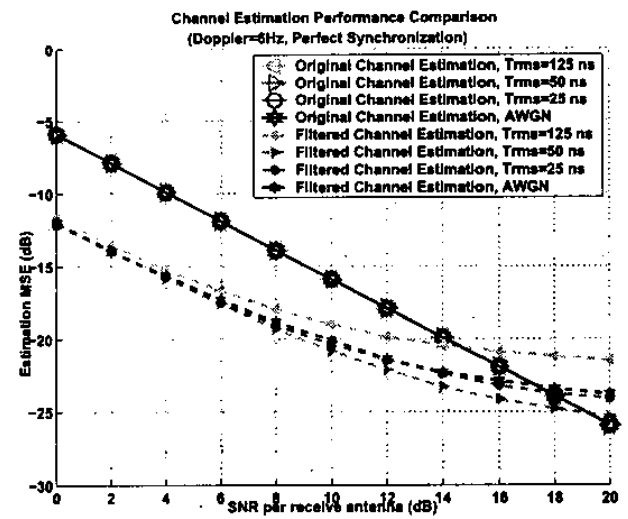

Fig. 5. Channel estimation MSE performance.

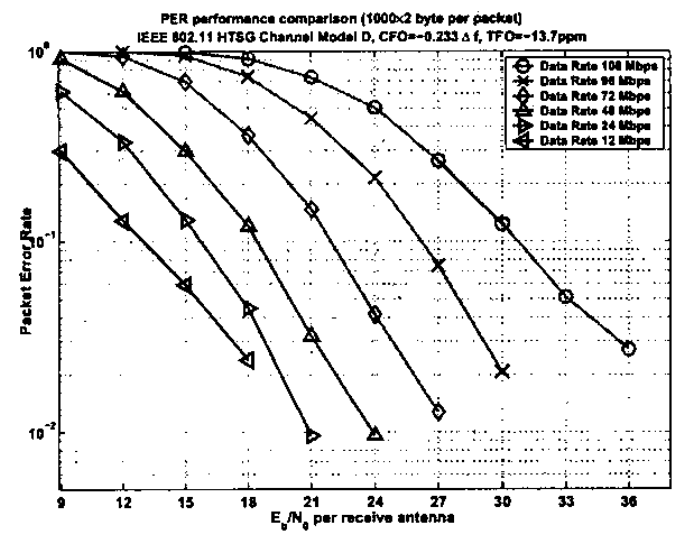

Fig. 6. PER performance of the proposed MIMO OFDM receiver.

at $-13.7 \mathrm{ppm}$ according to TGn comparison criteria [7]. The very small Doppler effect $(6 \mathrm{~Hz})$ is neglected for simplicity.

From the simulation results, the PER performance of the system running at the highest data rate reaches $10^{-1}$ at an $E_{b} / N_{0}$ of about $30 \mathrm{~dB}$. With the help of better outer coding, such as low-density product coding (LDPC), we can further improve the system performance to satisfy the functional requirement of the IEEE 802.11 task group $n$.

\section{CONCLUSION}

In this paper, we propose a MIMO OFDM baseband inner transceiver design for the next-generation high-throughput wireless LAN using two transmitting antennas and two receiving antennas. Algorithms for timing and frequency synchronization, tracking, channel estimation, and MIMO detection are designed and implemented. Finally, the PER performance of the receiver is presented and the results indicate that the proposed receiver is capable of delivering reliable transmission with double the data rate achieved by the current IEEE 802.11 a standard.

\section{REFERENCES}

[1] G. J. Foschini and M. J. Gans, "On limits of wireless communications in a fading environment using multiple antenna," Wireless Personal Commun., vol. 6, pp. 311335, Mar. 1998.

[2] P.W. Wolniansky, G.J. Foschini, G.D. Golden, and R.A. Valenzuela, "V-BLAST: an architecture for realizing very high data rates over the rich-scattering wireless channel," in URSI International Symposium on Signals, Systems, and Electronics, Oct. 1998, pp. 295-300.

[3] Siavash M. Alamouti, "A simple transmit diversity technique for wireless communications," IEEE J. Select. Areas Commun., vol. 16, pp. 1451-1458, Aug. 1998.

[4] A. N. Mody and G. L. Stuber, "Receiver implementation for a MIMO OFDM system," in Globecom, 2002, pp. 716-720.

[5] Pei-Yun Tsai, Hsin-Yu Kang, and Tzi-Dar Chiueh, "Joint weighted least squares estimation of frequency and timing offset for OFDM systems over fading channels," in Vehicular Technology Conference, April 2003, vol. 4, pp. 2543-2547.

[6] Pei-Yun Tsai and Tzi-Dar Chiueh, "Frequency-domain interpolation-based channel estimation in pilot-aided OFDM systems," IEEE VTC Spring, May 2004.

[7] IEEE 802.11 TGn, "Comparison criteria," DCN: 11-030814-rl 6 